\title{
Hipparcos astrometric orbits for two brown dwarf companions: HD 38529 and HD 168443
}

\author{
S. Reffert and A. Quirrenbach
}

Sterrewacht Leiden, Niels Bohrweg 2, 2333 CA Leiden, The Netherlands

e-mail: [sabine; quirrenbach]@strw.leidenuniv.nl

Received 30 November 2005 / Accepted 6 December 2005

\section{ABSTRACT}

Context. HD 38529 and HD 168443 have previously been identified as systems with two substellar companion candidates using precise radial velocity measurements.

Aims. We want to further constrain their orbits and the nature of the outer companions.

Methods. We fit astrometric orbits of the outer substellar companions in the two systems to the Hipparcos Intermediate Astrometric Data.

Results. The fit constrains all possible solutions to a small region in the parameter space of the two missing orbital parameters (inclination $i$ and ascending node $\Omega$ ). This can be interpreted as a possible real detection of the astrometric signatures of the companions in the Hipparcos data, although there is still a $14-18 \%$ chance that the signal is not detectable in the data, according to an F-test. However, even in the case of a non-detection of the companion signal in the astrometric data, the knowledge of the spectroscopic orbital parameters enables us to place tight constraints on these two missing parameters, so that the astrometric orbit is fully determined (with confidence levels of around $80 \%$ for HD 38529, 95\% for HD 168443). Inclinations derived from these astrometric fits enable us to calculate masses for the substellar companions rather than lower or upper limits. The best fit solution for HD $38529,(i, \Omega)=\left(160^{\circ}, 52^{\circ}\right)$, yields a mass of $37_{-19}^{+36} M_{\text {Jup }}$ for the outer companion. For HD 168443 , we derive best fit parameters of $(i, \Omega)=\left(150^{\circ}, 19^{\circ}\right)$, which imply a companion mass of $34 \pm 12 M_{\text {Jup }}$.

Conclusions. The outer companions in both systems are thus brown dwarfs.

Key words. stars: individual: HD 38529, HD 168443 - stars: low-mass, brown dwarfs - planetary systems - astrometry

\section{Introduction}

Although to date more than 150 extrasolar planets have been discovered, mainly with the radial velocity (RV) technique, only very few of them have established masses in the substellar regime. This is due to the fact that for modeling the observed radial velocity curves, only the mass function is needed. Together with a mass estimate for the primary, this yields the lower mass limit $m_{2} \cdot \sin i$, where $m_{2}$ is the mass of the companion and $i$ is the inclination of the orbit. In order to unambiguously determine the mass of the planetary companion candidate, additional information, for example from a photometric transit (restricted to systems with orbital inclinations close to $90^{\circ}$ ) or from an astrometric detection of the orbital motion is needed.

To date, only very few planetary companion masses are known unambiguously. Besides the planets discovered in transit searches, these are the planets in the HD 209458 and HD 149026 systems, which have been found to transit after they had been discovered via radial velocities, and the outer companion in the G1876 system. Gl 876b is so far the only planet which has an astrometrically determined mass, using the Fine Guidance Sensors of the Hubble Space Telescope (Benedict et al. 2002).
Astrometric observations by the Hipparcos satellite were found to be in general not accurate enough to detect astrometric signatures of extrasolar planets (Pourbaix 2001; Pourbaix \& Arenou 2001). However, even a non-detection of astrometric motion by Hipparcos can put stringent upper limits on companion masses, and thus firmly establish the substellar nature of an object. This approach has been followed first by Perryman et al. (1996), who determined upper mass limits in the substellar regime for the companions to $47 \mathrm{UMa}\left(22 M_{\mathrm{Jup}}\right)$ and $70 \mathrm{Vir}$ $\left(65 M_{\text {Jup }}\right)$. For the close-in planet around $51 \mathrm{Peg}$, no meaningful upper mass limit could be deduced. Similarly, Mazeh et al. (1999) have found the astrometric signature of the outermost planet in the $v$ And system, albeit with low confidence, yielding an upper an upper mass limit (of about $15 M_{\text {Jup }}$ ) as well. For the companion to $\iota$ Dra, Frink et al. (2002) derived an upper mass limit of $45 M_{\text {Jup }}$ from the non-detection of astrometric orbital motion in the Hipparcos data. Furthermore, an upper mass limit of $30 M_{\text {Jup }}$ was established for the companion to $\rho^{1} \mathrm{Cnc}$ by McGrath et al. (2002), based on observations with the HST Fine Guidance Sensors.

Here we derive masses for the outer RV detected substellar companion candidates around HD 38529 and HD 168443 from the Hipparcos Intermediate Astrometric Data. Both companions turn out to be brown dwarfs rather than planets, which is 
Table 1. Stellar characteristics and spectroscopic orbital elements for the outer companions in the HD 38529 and HD 168443 systems, quoted from the original discovery papers (Fischer et al. 2003; and Marcy et al. 2001, respectively). These elements are adopted without modifications for the astrometric solutions.

\begin{tabular}{lrr}
\hline \hline & HD 38529 & HD 168443 \\
\hline spectral type & G4 IV & G6 IV \\
mass $m_{1}\left[M_{\odot}\right]$ & 1.39 & 1.01 \\
\hline & HD 38529c & HD 168443c \\
\hline Period $P$ [days] & 2174.3 & 1770 \\
& \pm 30.0 & \pm 25 \\
Periastron time $T_{0}[\mathrm{JD}]$ & 2450073.8 & 2450250.6 \\
& \pm 35.0 & \pm 18 \\
Eccentricity $e$ & 0.36 & 0.20 \\
& \pm 0.05 & \pm 0.01 \\
Longitude of periastron $\omega\left[^{\circ}\right]$ & 14.7 & 62.9 \\
& \pm 10.0 & \pm 3.2 \\
mass function $f(m)\left[M_{\odot}\right]$ & $9.1 \times 10^{-7}$ & $4.2 \times 10^{-6}$ \\
\hline$m_{2} \cdot \sin i\left[M_{\text {Jup }}\right]$ & 12.79 & 17.15 \\
Semi-major axis $[\mathrm{AU}]$ & 3.68 & 2.87 \\
\hline
\end{tabular}

not surprising given their rather large lower mass limits derived from the radial velocities.

\section{Spectroscopic results}

\section{1. $H D 38529$}

HD 38529 (HIP 27253) has two low-mass companions that have been detected by measurements of precise radial velocities. The inner one, HD 38529b, was announced by Fischer et al. (2001) with a period of 14.3 days, whereas elements for the outer companion were published by Fischer et al. (2003), with a period of roughly six years. With a primary mass of $1.39 M_{\odot}$, derived minimum companion masses are $0.78 M_{\text {Jup }}$ for the inner companion and $12.70 M_{\text {Jup }}$ for the outer companion, the latter of which is most likely a brown dwarf. For convenience, the derived spectroscopic orbital parameters for the outer companion are listed in Table 1. The outer companion, rather massive and in a wide orbit, is very favorable for an astrometric detection. Indeed, the expected astrometric signature for the outer companion is at least 1.5 mas (depending on the unknown inclination), whereas the inner companion has a minimum astrometric signature of only $3 \mu$ as. Thus, the inner companion is not taken into account at all in the astrometric orbit; its effect is completely negligible.

\section{2. $H D 168443$}

HD 168443 (HIP 89844) also hosts a system of two known substellar companions. The inner one was discovered by Marcy et al. (1999), who noted already the likely presence of an outer companion. When more spectra were available and the orbit closed, Marcy et al. (2001) provided orbital elements for the outer companion as well and analyzed the system in detail. Udry et al. (2002) also published orbital elements for the two substellar companions in HD 168443, which are in good agreement with the Marcy et al. (2001) elements. The mass of the slightly evolved (G6 IV) primary star is assumed to be $1.01 M_{\odot}$, in accordance with Marcy et al. (2001) and Gonzalez et al. (2001). Derived minimum companion masses amount to $7.73 M_{\text {Jup }}$ for the inner companion and $17.15 M_{\text {Jup }}$ for the outer companion, the latter of which thus is a brown dwarf candidate. The corresponding minimum astrometric signatures are 0.1 mas and 2.4 mas, respectively. Again, the contribution of the inner companion is completely neglected in the following astrometric analysis of HD 168443.

\section{Astrometric results}

\subsection{Hipparcos astrometric orbit}

The Hipparcos Intermediate Astrometric Data, which were published along with the Hipparcos Catalogue (ESA 1997), offer the possibility to improve the solution in the Hipparcos Catalogue by modifying the underlying astrometric model for a particular star based on additional external observations and new insight. The individual one-dimensional Hipparcos measurements (abscissae) are given as abscissae residuals, which can be minimized by a standard $\chi^{2}$ fit based on a new model for a particular star. Details on how that can be achieved can be found in van Leeuwen \& Evans (1998).

The solution that was adopted for the Hipparcos Catalogue was the five parameter standard solution for HD 168443, while for HD 38529 a seven parameter solution was chosen, allowing for acceleration in the proper motions. This might already serve as a first hint that a companion to HD 38529 is present in the Hipparcos data, with a period likely larger than the Hipparcos mission lifetime of about 3 years. For HD 38529, 39 abscissa measurements are available, while for HD 168443 there are 34 abscissae. Of the 39 abscissae for HD 38529, one was only derived by the NDAC consortium and not by FAST. However, it was rejected for the solution given in the Hipparcos Catalogue (but is retained in our analysis below). Likewise, for HD 168443 there are three spacecraft orbits for which only the NDAC consortium produced a result, and one orbit where there is only an abscissa from the FAST consortium. Furthermore, one FAST abscissa was rejected for the standard solution in the Hipparcos Catalogue. We decided to also reject this abscissa in our solution, since the abscissa is off by about $10 \sigma$ from the NDAC abscissa for the same orbit, and the absolute value of the abscissa residual is at least an order of magnitude larger than all other abscissa residuals, indicating a likely flaw in the FAST reduction of that abscissa.

We properly decorrelated and weighted all available individual abscissa measurements for the two stars following the procedure outlined in van Leeuwen \& Evans (1998). We then fitted an orbital solution to these data, keeping the spectroscopic parameters from Table 1 fixed. There were seven free parameters in the fit: two unknown orbital parameters (inclination $i$ and ascending node $\Omega$ ), and five astrometric parameters (mean positions and proper motions in right ascension and declination, respectively, and the parallax). For the astrometric parameters, differential offsets from the best fit parameters 
Table 2. The remaining orbital elements $i$ and $\Omega$ for HD 38529 and HD 168443, as obtained from our astrometric fit using the Hipparcos Intermediate Astrometric Data. Corrections to the standard five astrometric parameters in Hipparcos, derived from the same fit, are also given, as well as the derived companion masses based on our best fit inclinations.

\begin{tabular}{|c|c|c|}
\hline & HD 38529c & HD $168443 c$ \\
\hline $\begin{array}{l}\text { Inclination } i\left[^{\circ}\right] \\
\quad \text { uncertainty in } \chi^{2} \text { fit } \\
\text { uncertainty due to RV elements }\end{array}$ & $\begin{array}{r}160 \\
+7 /-23 \\
\pm 7\end{array}$ & $\begin{array}{r}150 \\
+8 /-20 \\
\pm 0.5\end{array}$ \\
\hline $\begin{array}{l}\text { Ascending Node } \Omega\left[^{\circ}\right] \\
\text { uncertainty in } \chi^{2} \text { fit } \\
\text { uncertainty due to RV elements }\end{array}$ & $\begin{array}{r}52 \\
+24 /-23 \\
\pm 17\end{array}$ & $\begin{array}{r}19 \\
+21 /-24 \\
\pm 7\end{array}$ \\
\hline $\begin{array}{l}\Delta \alpha[\mathrm{mas}] \\
\Delta \delta[\mathrm{mas}] \\
\Delta \pi[\mathrm{mas}] \\
\Delta \mu_{\alpha *}\left[\mathrm{mas} \mathrm{yr}^{-1}\right] \\
\Delta \mu_{\delta}\left[\mathrm{mas} \mathrm{yr}^{-1}\right]\end{array}$ & $\begin{array}{r}-0.05 \\
-0.46 \\
-1.81 \\
2.14 \\
1.32\end{array}$ & $\begin{array}{r}0.69 \\
-0.56 \\
0.67 \\
-0.80 \\
-1.04\end{array}$ \\
\hline$m_{2}\left[M_{\text {Jup }}\right]$ & $\begin{array}{r}37 \\
+36 /-19 \\
\end{array}$ & $\begin{array}{r}34 \\
\pm 12 \\
\end{array}$ \\
\hline $\begin{array}{l}\text { reduced } \chi^{2} \text { (our fit) } \\
\text { reduced } \chi^{2} \text { (Hipparcos Catalogue) } \\
\text { F test probability [\%] }\end{array}$ & $\begin{array}{c}1.57 \\
1.75^{a} \\
18\end{array}$ & $\begin{array}{r}0.99 \\
1.16 \\
14\end{array}$ \\
\hline
\end{tabular}

${ }^{a}$ For better comparison with our result, the given $\chi^{2}$ value corresponds to the 5 parameter solution in Hipparcos including all available abscissae.

in the Hipparcos Catalogue were used. This is in contrast to Frink (2003), where the five standard astrometric parameters were kept fixed.

Our best fit parameters are listed in Table 2. The derived inclinations imply a mass of $37_{-19}^{+36} M_{\text {Jup }}$ for HD 38529c, and $34 \pm 12 M_{\text {Jup }}$ for HD $168443 \mathrm{c}$, placing both of them clearly into the brown dwarf regime. If the orbits in both systems were coplanar, the derived inclinations would yield a mass of $2.3 M_{\text {Jup }}$ for HD $38529 \mathrm{~b}$, and a mass of $15 M_{\text {Jup }}$ for HD $168443 \mathrm{~b}$. The assumption of co-planarity might not be valid for the HD 38529 system, which is dynamically stable even for high mutual inclinations of the two companions (Kiseleva-Eggleton et al. 2002). In contrast to that, the HD 168443 system seems to be dynamically stable only if there is no mutual inclination between the two companions (Marcy et al. 2001), so that the assumption of co-planarity might be justified for that system.

\subsection{Significance of the astrometric solutions}

The above fits are likely real detections of the astrometric signatures of the outer companions rather than a derivation of the upper mass limit. In Fig. 1 we have plotted contours of constant $\chi^{2}$, denoting confidence regions with probabilities of $68.3 \%(1 \sigma), 90 \%$ and $95 \%$ for both parameters $i$ and $\Omega$ jointly. In the case of HD 168443, even the outer $95 \%$ confidence level contour spans less than half of the total $(i, \Omega)$ parameter space, so that - with a probability of $95 \%$ - the astrometric orbit is really detected in the data. For HD 38529, the significance level for the detection of the astrometric orbit is somewhat less; the outer contour in Fig. 1, indicating again the $95 \%$ confidence level for both parameters jointly, extends over the full range of possible $\Omega$ values. Also, there is a small ambiguity in the orientation of the orbit, even with the $68.3 \%$ confidence level contours: the most likely minimum is at $(i, \Omega)=\left(160^{\circ}, 52^{\circ}\right)$, but another local minimum in the $\chi^{2}$ contour map is found at $\left(180^{\circ}-i, 360^{\circ}-\Omega\right)$, which corresponds to an orbit with the same geometry but the opposite sense of revolution. If one were to assign a probability for the astrometric orbit to be real, it would be around $80 \%$; this confidence level is not indicated in the figure, but it would make the accepted parameter space comparable in size to the one accepted by the $95 \%$ contour for HD 168443 . We conclude that the astrometric orbits are detected in the Hipparcos Intermediate Astrometric Data with confidence levels of around $80 \%$ for HD 38529 and $95 \%$ for HD 168443.

A secondary indicator is provided by the F-test, which measures the improvement of the $\chi^{2}$ fit when introducing new parameters as compared to the original fit (see also Pourbaix $\&$ Arenou 2001). With a probability of $18 \%$ in the case of HD 38529 and $14 \%$ in the case of HD 168443, the astrometric signal is not present in the data, according to an F-test. However, we note that a simple test like the F-test cannot take into account the density or steepness of the $\chi^{2}$ contours, nor outside constraints as come from the radial velocity measurements, so that its use is limited for our purposes. It does tell us however that introducing a number of additional parameters indeed significantly improves the fit, to within the given error probabilities.

However, we would like to stress again that even with F-test probabilities of $14-18 \%$ that the astrometric signal is not present in the Hipparcos data, one can see from the effect of the ascending node on the $\chi^{2}$ in Fig. 1 that the Hipparcos data indeed carry the signature of the companions: the $\chi^{2}$ value is rather sensitive to the exact choice of the ascending node, especially for HD 168443c. If the astrometric signal was not present in the data, then the ascending node should have a negligible effect on the $\chi^{2}$, since it does not change the amplitude of the signal (as opposed to the inclination).

Another issue to take into account are the uncertainties of the five spectroscopic parameters, which have been held fixed in our astrometric solution but also carry uncertainties. In order to assess how our results are affected by these uncertainties, we generated 1000 Monte-Carlo sets of spectroscopic parameters that are consistent with the RV best fit solutions to within the errors. We then derived best fit $(i, \Omega)$ combinations in the same way as described above. The results are overplotted as small dots in Fig. 1. The narrow region that is outlined by these solutions clearly demonstrates the robustness of the result, especially for HD 168443, where the inferred inclination does not depend on the precise values of the spectroscopic parameters. For HD 38529c, 5\% of the generated sets of spectroscopic parameters actually yield a best fit orbit with the opposite orientation as the original solution, as was already indicated by the second local minimum in the $\chi^{2}$ maps.

We also tried to assess the robustness of our astrometric fits by using either only FAST or NDAC abscissae, and 

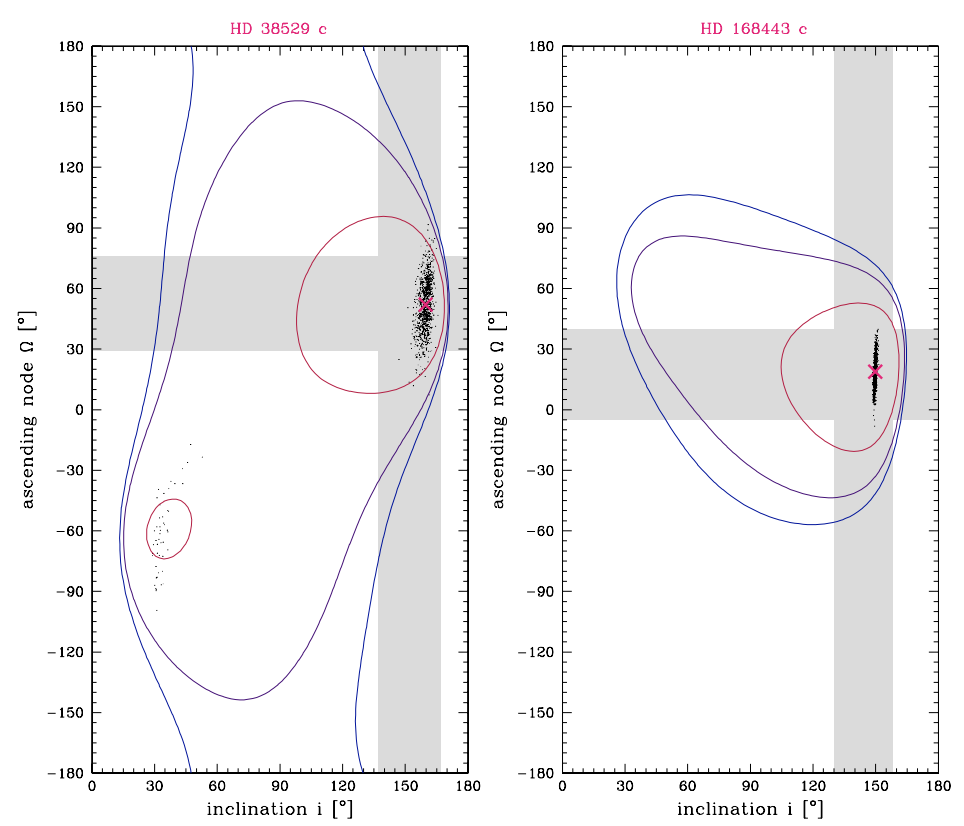

Fig. 1. $\chi^{2}$ contours for fitting a substellar companion with fixed spectroscopic parameters to the Hipparcos Intermediate Astrometric Data of HD 38529 (left panel) and HD 168443 (right panel). The inclination $i$ and ascending node $\Omega$ were free parameters in the fit, as were corrections to the standard five astrometric parameters in the Hipparcos Catalogue. The contours correspond to the confidence levels with probabilities of $68.3 \%$ $(1 \sigma), 90 \%$ and $95 \%$ as appropriate for two parameters; the $1 \sigma$ confidence intervals for only one parameter at a time are shaded in gray. The best fit solution is indicated with a cross. The small dots in each panel correspond to best fit solutions where errors in the spectroscopic parameters were taken into account. Note the small region in the parameter space taken up by the $\chi^{2}$ contours, placing significant constraints on the astrometric orbits, especially for HD $168443 \mathrm{c}$. by keeping the proper motions fixed to a value from another catalog that might be a better representation of the real longterm motion through space, rather than the instantaneous Hipparcos motion which might be affected by orbital motion. Neither of these modifications changed our best fit astrometric parameters significantly.

\section{Discussion}

We have determined the masses of the outer companions in the HD 38529 and HD 168443 systems as $37_{-19}^{+36} M_{\text {Jup }}$ and $34 \pm 12 M_{\text {Jup }}$, respectively, from an orbital fit of the astrometric signature to the Hipparcos Intermediate Data. For HD 168443c, this mass is very close to the upper limit of $42.8 M_{\text {Jup }}$ derived by Marcy et al. (2001), who also used the Hipparcos Intermediate Astrometric Data. The results are in very good agreement with each other, and it is no coincidence that the actual mass of the companion is so close to its upper limit set by Hipparcos: if this was not the case, we either had not been able to derive any constraints on the astrometric orbit at all, or it would have been so obvious that the star would have been solved as a binary star in the Hipparcos Catalogue itself. Only if the actual mass of the companion is close to the detection limit in the Hipparcos data can one expect significant improvement by using the spectroscopic parameters as additional constraints in the astrometric fit.

The detection of the astrometric orbit of substellar companions does not only provide the true masses without the $\sin i$ ambiguity, but it also determines the orientation of the orbit in space. This could be helpful in attempts to directly detect these companions, since accurate predictions of angular separation and position angle can be made.

In the near future, it should become possible to measure the orientation of stellar rotation axes in space with optical long-baseline interferometry (Quirrenbach 2004), and thus to determine whether HD 38529c and HD 168443c orbit in the equatorial plane of their parent stars or not. This will provide further constraints on the formation and early dynamical evolution of these systems.

\section{References}

Benedict, G. F., McArthur, B. E., Forveille, T., et al. 2002, ApJ, 581, L115

Brown, T. M., Charbonneau, D., Gilliland, R. L., Noyes, R. W., \& Burrows, A. 2001, ApJ, 552, 699

Charbonneau, D., Brown, T. M., Latham, D. W., \& Mayor, M. 2000, ApJ, 529, L45

ESA 1997, The Hipparcos and Tycho Catalogues, ESA SP-1200

Fischer, D. A., Marcy, G .W., Butler, R. P., et al. 2001, ApJ, 551, 1107

Fischer, D. A., Marcy, G. W., Butler, R. P., et al. 2003, ApJ, 586, 1394

Frink, S. 2003, in Proc. Conf. on Towards Other Earths: DARWIN/TPF and the Search for Extrasolar Terrestrial Planets, ed. M. Fridlund, \& T. Henning, ESA SP-539, 413

Frink, S., Mitchell, D. S., Quirrenbach, A., et al. 2002, ApJ, 576, 478

Gonzalez, G., Laws, C., Tyagi, S., \& Reddy, B. E. 2001, AJ, 121, 432

Henry, G. W., Marcy, G. W., Butler, R. P., \& Vogt, S. S. 2000, ApJ, 529, L41

Marcy, G. W., Butler, R. P., Vogt, S. S., Fischer, D., \& Liu, M. C. 1999, ApJ, 520, 239

Marcy, G. W., Butler, R. P., Vogt, S. S., et al. 2001, ApJ, 555, 418

Mazeh, T., Zucker, S., dalla Torre, A., \& van Leeuwen, F. 1999, A\&A, 522, L149

McGrath, M. A., Nelan, E., Black, D. C., et al. 2002, ApJ, 564, L27

Kiseleva-Eggleton, L, Bois, E., Rambaux, N., \& Dvorak, R., ApJ, 587, L145

Perryman, M. A. C., Lindegren, L., Arenou, F., et al. 1996, A\&A, 310, L21

Pourbaix, D. 2001, A\&A, 369, L22

Pourbaix, D., \& Arenou, F. 2001, A\&A, 372, 935

Quirrenbach, A. 2004, in New frontiers in stellar interferometry, ed. W. A. Traub, SPIE, 5491, 146

Udry, S., Mayor, M., Naef, D., et al. 2002, A\&A, 390, 267

van Leeuwen, F., \& Evans, D. W. 1998, A\&AS, 130, 157 